\title{
Majority-vote model on hyperbolic lattices
}

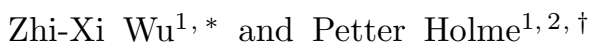 \\ ${ }^{1}$ Department of Physics, Umeå University, 90187 Umeå, Sweden \\ ${ }^{2}$ Department of Energy Science, Sungkyunkwan University, Suwon 440-746, Korea
}

\begin{abstract}
We study the critical properties of a non-equilibrium statistical model, the majority-vote model, on heptagonal and dual heptagonal lattices. Such lattices have the special feature that they only can be embedded in negatively curved surfaces. We find, by using Monte Carlo simulations and finite-size analysis, that the critical exponents $1 / \nu, \beta / \nu$ and $\gamma / \nu$ are different from those of the majority-vote model on regular lattices with periodic boundary condition, which belongs to the same universality class as the equilibrium Ising model. The exponents are also from those of the Ising model on a hyperbolic lattice. We argue that the disagreement is caused by the effective dimensionality of the hyperbolic lattices. By comparative studies, we find that the critical exponents of the majority-vote model on hyperbolic lattices satisfy the hyperscaling relation $2 \beta / \nu+\gamma / \nu=D_{\text {eff }}$, where $D_{\text {eff }}$ is an effective dimension of the lattice. We also investigate the effect of boundary nodes on the ordering process of the model.
\end{abstract}

PACS numbers: 05.50.+q, 02.40.Ky, 64.60.Cn, 05.70.Ln

\section{INTRODUCTION}

Recently, there has been a growing interest the critical behavior of statistical-physics models on curved surfaces - ranging from spin models, such as the ferromagnetic Ising model 1, 2, 3, 4], the $X Y$ model [5, 6], the Heisenberg model [7], the $q$-state clock models [8, 9], to other traditional models, such as percolation [10], diffusion [11], etc. One reason for this interest is that many newly discovered soft materials (e.g., carbon nanotubes) show a negatively curved structure in the nanoscale [12]. One peculiar feature of negatively curved surfaces is that their boundary is a finite fraction of the whole geometry [13]. This structure has been verified having a nontrivial impact on the critical behavior of many models of statistical physics. For example, in the context of the Ising model, significant shifts in static and dynamic critical exponents toward the mean-field values were noticed [1, 2]; small-sized ferromagnetic domains were observed to exist at temperatures far greater than the critical temperature [4]; An apparent zero-temperature orientational glass transition in the $X Y$ spin model on a negatively curved surface was recently demonstrated [6].

These findings motivate us to investigate another spin models on negatively curved surfaces. The majority-vote model [14, 15, 16, 17] is a simple non-equilibrium model exhibiting up-down symmetry that has been argued to belong to the universality class of the equilibrium Ising model [18]. Oliveira first verified this conjecture on a square lattice with periodic boundary conditions (i.e., a torus) [14]. Subsequently, the majority-vote model has been investigated on regular lattices (with dimension larger than two) [16, 17, 19, 20], random lattice [21], directed or undirected random graphs [22, 23], small world

\footnotetext{
*Electronic address: zhi-xi.wu@physics.umu.se
}

${ }^{\dagger}$ Electronic address: petter.holme@physics.umu.se networks [24], and scale-free networks [25], etc. Very recently, it has been found that the critical behavior of the majority-vote model on square lattice is also independent of transition rates (e.g., the Glauber or Metropolis rates) [19]. It has also been observed that the majorityvote models defined on different complex networks belong to different universality classes [22, 23, 24, 25].

Our goal in this contribution is therefore to identify the critical behavior of the majority-vote model when the underlying lattice is embedded in a hyperbolic surface, in particular the heptagonal and the dual heptagonal lattices, and investigate if such an interaction structure is capable of modifying the critical exponents. To this end, we use Monte Carlo (MC) simulations and standard finite-size scaling techniques to determine the critical noise parameter $q_{c}$ (the main control parameter of the majority-vote model, as well as the critical exponents $1 / \nu, \beta / \nu$ and $\gamma / \nu$. Our numerical results suggest that the critical exponents, in the stationary state, are different from those of the Ising model confined to regular and hyperbolic lattices.

In the following Sec. III, we define our model, describe the quantities we measure and the computational details. In Sec. III, we present our numerical results and analysis. Finally, we summarize and contextualize the observations in Sec. IV.

\section{MODEL AND SIMULATION}

\section{A. Majority-vote model}

Following Refs. [14, 15, 16, 17, 21, 22, 23, 24], the majority-vote model with noise is defined by a set of spin variables $\left\{\sigma_{i}\right\}$, where each spin is associated to one node of the heptagonal lattice and can take the values \pm 1 . The system evolves as follows: For each spin $i$, we first determine the majority spin of $i$ 's neighborhood. With probability $q$ the $i$ takes the opposite sign of the majority 
spin, otherwise it takes the same spin as the majority spin. The probability $q$ is called the noise parameter and plays the same role of temperature in equilibrium spin systems. In terms of $q$, the probability of a single-spinflip is given by

$$
w\left(\sigma_{i}\right)=\frac{1}{2}\left[1-(1-2 q) \sigma_{i} S\left(\sum_{j \in \Omega_{i}} \sigma_{j}\right)\right],
$$

where $S(x)=\operatorname{sgn}(x)$ if $x \neq 0$ and $S(0)=0$, and the summation is over all the neighboring spins of the focal site $i$. The transition probability (1) satisfies up-down symmetry under the change of signs of the spins in the neighborhood of $i$. In the limit of zero noise, the majority-vote model is identical to the ferromagnetic Ising model at zero temperature [14, 24].

\section{B. Heptagonal lattice and dual heptagonal lattice}

Figure 1 shows two examples of the heptagonal lattice and the dual heptagonal lattice. One peculiar property of the heptagonal lattice is that, if we consider the innermost heptagon as the level one, then the number of nodes of a heptagonal lattice with level $l$ can be calculated by using the formulation [4, 9], $N(l)=$ $1+\frac{15}{\sqrt{5}} \sum_{j=1}^{l}\left[\left(\frac{3+\sqrt{5}}{2}\right)^{j}-\left(\frac{3-\sqrt{5}}{2}\right)^{j}\right]$, which grows exponentially with level $l$. In other words, the ratio of the perimeter to the area of the lattice remains finite (about 0.62) in the thermodynamic limit $l \rightarrow \infty$ [4]. If we make map each heptagon in the heptagonal lattice to a node, and put a link between adjacent heptagons, then we get the dual heptagonal lattice [Fig. 1(b)]. Since these lattices can only be embedded in a hyperbolic surface with a constant negative curvature, they are also called hyperbolic lattices.
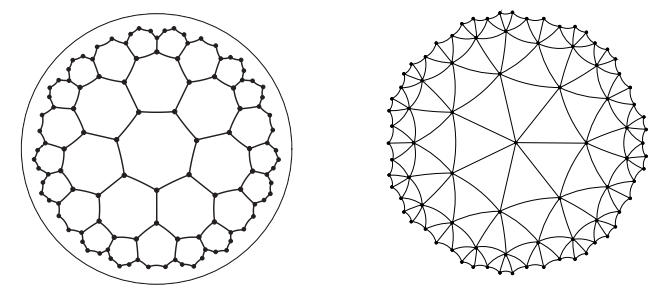

FIG. 1: A heptagonal lattice with level 3 (left), and the dual lattice with level 4 (right). For the heptagonal lattices with level $l=2,3,4,5,6,7$, the total number of nodes are $35,112,315,847,2240,5887$, respectively. For the dual lattice with level $l=3,4,5,6,7,8$, the total number of nodes are $29,85,232,617,1625,4264$, respectively.

\section{Measurements and finite size scaling}

In order to study the critical behavior of the model we consider the magnetization $M$, the susceptibility $\chi$, and the Binder's fourth-order cumulant $U$. These quantities are defined as follows:

$$
\begin{gathered}
M=\left\langle\langle m\rangle_{T}\right\rangle_{C}=\left\langle\left\langle\frac{1}{N}\left|\sum_{1}^{N} \sigma_{i}\right|\right\rangle_{T}\right\rangle_{C}, \\
\chi=N\left[\left\langle\left\langle m^{2}\right\rangle_{T}\right\rangle_{C}-\left\langle\langle m\rangle_{T}\right\rangle_{C}^{2}\right], \\
U=1-\frac{\left\langle\left\langle m^{4}\right\rangle_{T}\right\rangle_{C}}{3\left\langle\left\langle m^{2}\right\rangle_{T}\right\rangle_{C}^{2}},
\end{gathered}
$$

where $N$ is the total number of nodes of the hyperbolic lattice, $\langle\ldots\rangle_{T}$ denotes thermodynamics averages taken in the stationary regime, and $\langle\ldots\rangle_{C}$ stands for configurational averages.

The above quantities are functions of $q$ and $N$, in the critical region, we expect the following finite-size scaling relations

$$
\begin{gathered}
M(N, q)=N^{-\beta / \nu} \tilde{M}\left(N^{1 / \nu} \varepsilon\right) \\
\chi(N, q)=N^{\gamma / \nu} \tilde{\chi}\left(N^{1 / \nu} \varepsilon\right) \\
U^{\prime}(N, q)=N^{1 / \nu} \tilde{U}^{\prime}\left(N^{1 / \nu} \varepsilon\right)
\end{gathered}
$$

where $\varepsilon=q-q_{c}$ and $U^{\prime}$ is the derivative of Binder's fourth-order cumulant with respect to the noise. By the standard finite-size scaling approach [27], we assume scaling functions $\tilde{M}, \tilde{\chi}$, and $\tilde{U}$ that are continuous and differentiable in the vicinity of the critical noise $q_{c}$. From the size dependence of $M$ and $\chi$ we can obtain the exponents $\beta / \nu$ and $\gamma / \nu$, respectively. One alternative way to detect $\gamma / \nu$, since it also scales as $N^{\gamma / \nu}$ [27], is by plotting the maximum value of the susceptibility versus $N$.

\section{Computational method}

We implement our MC simulations on the hyperbolic lattices with various system sizes starting with all spins up and going from low noise to high noise. It has been pointed out that this method can reduce the relaxation time considerably, especially in the low noise limit, compared to starting with spins randomly oriented up or down 26]. For each given $q$, we simulated systems of size $N=112,315,847,2240,5887$ for the heptagonal lattice, and $N=85,232,617,1625,4264$ for the dual heptagonal lattice. For the sake of comparison, we also studied the majority-vote on square lattice with free boundary condition. In all simulations, we first wait $90000 \mathrm{MC}$ steps to let the system attain stationary state (in the high noise case, this number reduces to 50000 ), and then ran another $30000 \mathrm{MC}$ steps to get the average values. One MC step contains a sweep of the spins in a random sequence. After every tenth MC steps, we reshuffle the random sequence. Each data point presented below are averages over 500, 300 and 200 trials for $N<1000$, $1000<N<4000$, and $N>4000$, respectively. 

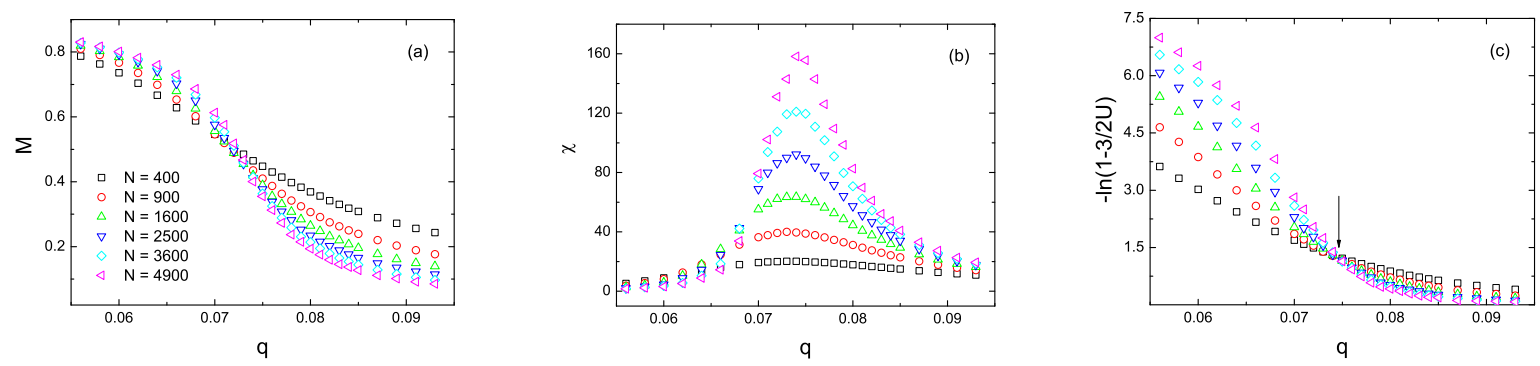

FIG. 2: (Color online) Majority-vote model on a two dimensional square lattice with a free boundary condition. Magnetization $M(\mathrm{a})$, susceptibility $\chi(\mathrm{b})$, and reduced fourth-order cumulant $U(\mathrm{c})$, as a function of the noise parameter $q$ for several values of the system size $N$. In (c), within the accuracy of the data, all curves intersect at $q_{c}=0.074(5)$.
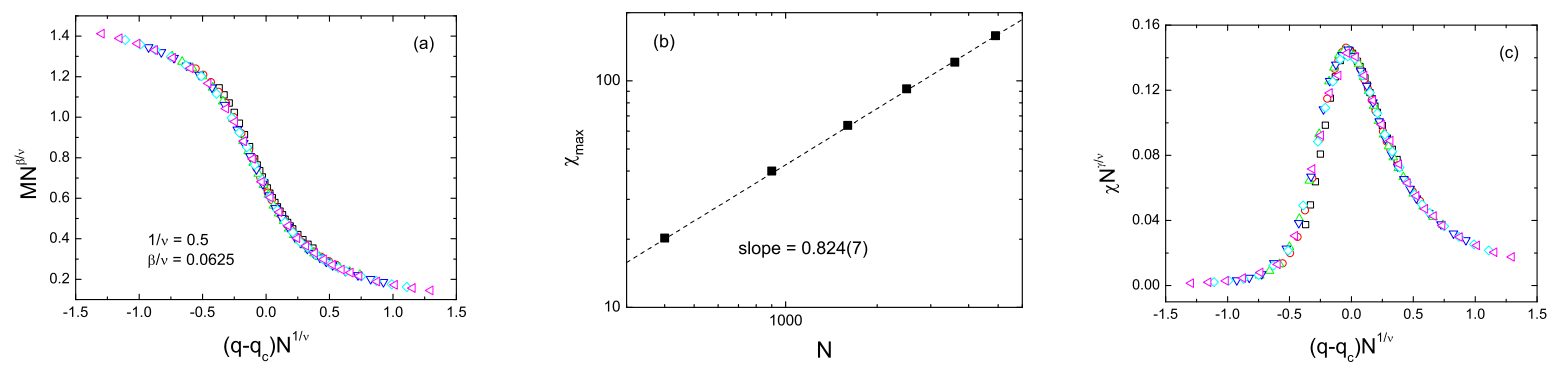

FIG. 3: (Color online) (a) Data collapse of the magnetization $M$ shown in Fig. 2(a). The exponents used for the data collapse are $1 / \nu=0.5$ and $\beta / \nu=0.0625$. (b) $\log -\log$ plot of the maximum of the susceptibility as a function of $N$. From it we estimate the critical exponent $\gamma / \nu=0.824(7)$ as the best fit of the data points. (c) Data collapse of the susceptibility shown in Fig. 2(c). The fitting exponents are $\beta / \nu=0.0625$ and $\beta / \nu=0.825$.

\section{NUMERICAL RESULTS AND FINITE SIZE SCALING ANALYSIS}

Previous investigations have shown that the majorityvote model undergoes a phase transition from an ordered to a disordered phase at a critical value of $q_{c}$. This critical value depends on the lattice topology [14, 15, 16]. It is worth noting that almost all previous studies assume a periodic boundary condition of the underlying topology. There are no previous results for the majority-vote model on regular lattice with a free boundary condition which is the case for the heptagonal and dual heptagonal lattices (Fig. 1). Therefore we proceed to investigate the majority-vote model on a square lattice with free boundary condition.

\section{A. Majority-vote model on square lattice with free boundary condition}

In Fig. 2 we present MC results of the majority-vote model on square lattice with free boundary condition and plot the magnetization, susceptibility, and reduced fourth-order cumulant as functions of the noise parameter $q$ for several values of $N$. As can be noticed in
Fig. 2(a) there is a phase transition from an ordered state $(M>0)$ to a disordered state $(M \approx 0)$. In Fig. $2(\mathrm{~b})$, the susceptibility $\chi$ reaches a maximum in the critical region for different system sizes which is another typical signature for the onset of criticality. The critical point $q_{c}$ can be detected from Fig. 2(c), where the curves of the reduced forth-order cumulant $U$ for different $N$ intersect with each other. We obtain $q_{c}=0.074(5)$, which agrees quite well with that for the majority-vote model on a square lattice with a periodic boundary condition $q_{c} \approx 0.075[14,15]$.

The results of Fig. 2(c) indicate that a vanishing fraction of boundary nodes do not change the critical value of $q$. In light of this point, we may expect the critical exponents to be the same. To check this assumption, we plot $M N^{\beta / \nu}$ versus $\left(q-q_{c}\right) N^{1 / \nu}$ in Fig [3(a) using the critical exponents $1 / \nu=0.5, \beta / \nu=0.0625$ of two dimensional Ising model. (Note that if we measure the critical exponents in terms of linear dimension $L$ instead of $N=L^{2}$, then $1 / \nu=1, \beta / \nu=0.125$.) The excellent collapse of the curves for six different system sizes corroborates the estimations for $q_{c}$ and the critical exponents $1 / \nu$ and $\beta / \nu$, and verifies the Ising universality class of the phase transition. On the other hand, the curves for $\chi N^{-\gamma / \nu}$ versus $\left(q-q_{c}\right) N^{1 / \nu}$ do not overlap in the critical 
region if $\gamma / \nu=0.875$ is used (results not shown), which hints that the free boundary condition induces a strong finite-size effect on the fluctuation of average magnetization (the critical exponent $\gamma / \nu$ ). For this reason, we use finite-size scaling by plotting $\chi_{\max }$ (the maximum value of the finite-size susceptibility) as a function of $N$. We get the slope $\gamma / \nu=0.824(7)$ of the best-fit line, as displayed in Fig. 3(b). The collapse of the curves for the rescaled susceptibility verifies this estimate [Fig. 3(c)].

\section{B. Majority-vote model on heptagonal lattice}

We now turn our attention to the majority-vote model on the heptagonal lattice. The simulation results for $M$, $\chi$, and $U$ as functions of the noise parameter $q$ for different $N$ are summarized in Fig 4. As was shown in Fig. 2(a), above the critical noise level, the magnetization disappears for larger system sizes, whereas it reaches a finite value in the subcritical region [Fig. 4(a)]. The susceptibility values in the critical region reach their maximal values [Fig. 4(b)], and the reduced fourth-order cumulants cross at the critical point, giving $q_{c}=0.034(8)$.

In order to study the universality class of the model, we proceed to measure the critical exponents. From Ref. [29], we know that the critical exponent $\nu$ can be obtained from the scaling behavior of certain thermodynamic derivatives with respect to $q$ (for example, the derivative of the cumulant and the logarithmic derivatives of $M$ ). In Fig. 5(a), we plot the maximum value of these derivatives as functions of system size on a log$\log$ scale. $1 / \nu$ obtained from these fits can be seen in Fig.5(a). From combining these two estimates, we obtain $1 / \nu=0.30(2)$. In Fig. 5(b), we display the $N$-dependence of the magnetization at $q_{c}$. From the slope of the dashed line, which corresponds to the best fit to the data points, we estimate the corresponding value of the critical exponent to $\beta / \nu=0.114(5)$. Using these values we proceed to plot $M N^{\beta / \nu}$ against $\left(q-q_{c}\right) N^{1 / \nu}$. From the finitesize scaling assumption that $\tilde{M}$ is a universal function, we should, for the correct choices of $1 / \nu$ and $\beta / \nu$, find a data collapse in the critical region [28]. In Fig. [5](c) we show that such a data collapse does indeed occur. In a similar way, we can determine the value of $\gamma / \nu$ by fitting the data for $\chi_{\max }$ as a function of $N$ in a $\log -\log$ scale, whose slope predicts $\gamma / \nu=0.721(7)[\mathrm{Fig}$. 5)(d)]. By plotting $\chi N^{\gamma / \nu}$ versus $\left(q-q_{c}\right) N^{1 / \nu}$ with $1 / \nu=0.3$ and $\gamma / \nu=0.722$, however, we only get good collapse for the curves in the supercritical region, i.e., $q>q_{c}$ [Fig. 5(e)]. In the subcritical region the curves deviate, suggesting an anomalous scaling behavior. This effect can also be seen in Fig. 3(d) for small system sizes. Since the boundary vanishes with size in Fig. 3, as does the deviation from the scaling collapse, but the boundary does not vanish in Fig. [5] and neither does the deviation, we conclude that the boundary is probably causing the anomalous scaling behavior.

\section{Majority-vote model on dual heptagonal lattice}

We proceed to investigating the majority-vote model on the dual heptagonal lattice. As above, we first present our numerical results for magnetization, susceptibility and reduced fourth-order cumulant as a function of the noise parameter (Fig. 6). The qualitative properties of these quantities as functions of $q$ are similar to the observations in Figs. 22 and 4. Also here, from the intersection of the curves in Fig. 6(c), we obtain $q_{c}=0.087(5)$. The critical exponents $1 / \nu, \beta / \nu$, and $\gamma / \nu$ are estimated in Figs. 7(a), (b) and (d) to 0.32(9), 0.093(6), and 0.761(9) respectively. The data collapse of the magnetization in Fig. 7(c) confirms these measurements. Also here these values do not give a good data collapse of $\chi$ in the region $q<q_{c}$.

Up to now, we obtained the critical exponents $1 / \nu$, $\beta / \nu$ and $\gamma / \nu$ for the majority-vote model on the heptagonal and dual heptagonal lattices. These exponents, and thus the universality classes, are different from the same model on square lattices. Interestingly, by checking the hyperscaling relation among the exponents [27]

$$
2 \beta / \nu+\gamma / \nu=D_{\text {eff }},
$$

we find that $D_{\text {eff }}=0.125+0.824(7)=0.949(7)$ for the square lattice with free boundary condition, $D_{\text {eff }}=$ $0.229+0.721(6)=0.950(6)$ for the heptagonal lattice, and $D_{\text {eff }}=0.187(2)+0.761(9)=0.949(1)$ for the dual heptagonal lattice. Without the finite-size effects mentioned above, these values are, we believe, consistent with 1. (If the scaling variable is chosen to be $L=N^{1 / 2}$, this means $D_{\text {eff }}=2$.) In terms of these values and taking the finite-size scaling effect into account, we propose that our critical exponents for the majority-vote model on negatively curved surface also satisfy a hyperscaling relation like the Rushbrooke and Josephson scaling laws $2 \beta+\gamma=\nu d=D_{\text {eff }}$, where $d$ is a dimension of the underlying lattice. Since the value $1 / \nu$ for both the heptagonal and dual heptagonal lattices are smaller than 0.5 , our results can be explained by an effective dimensionality of the two hyperbolic lattices greater than 2. (This is reasonable - the dimensionality of hyperbolic surfaces, embedded in an Euclidean geometry, is also larger than two.)

\section{Effect of boundary nodes}

From our numerical results, we know that the critical behaviors of the majority-vote model on the heptagonal and dual heptagonal lattices are different. One potential explanation comes from by the different topological structure of the boundary nodes. (The boundary nodes of the regular heptagonal lattice interacts less with the inner part of the surface compared with the dual heptagonal case.)

To explore this boundary effect, one straightforward way is to consider a (regular or dual) heptagonal lattice 

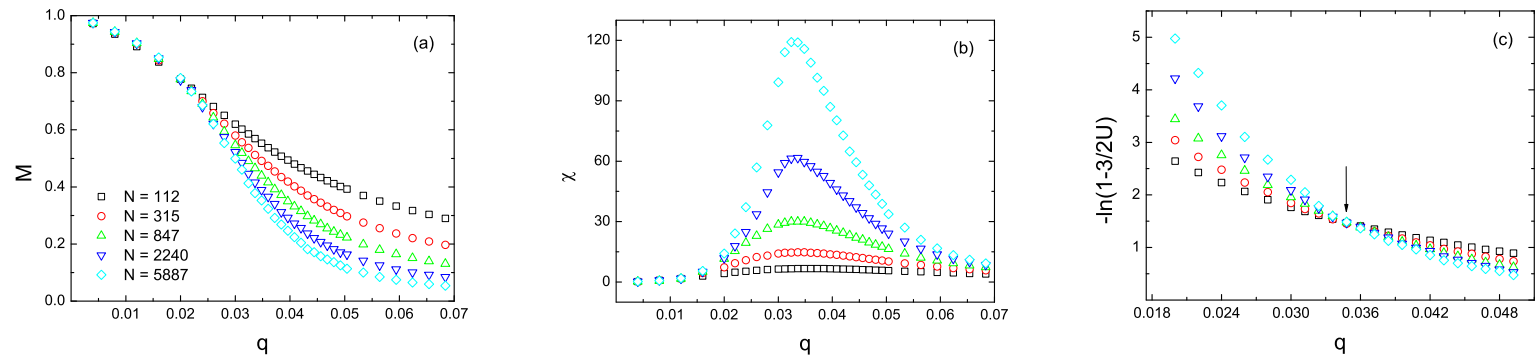

FIG. 4: (Color online) Majority-vote model on the heptagonal lattices. Magnetization $M$ (a), susceptibility $\chi$ (b), and reduced fourth-order cumulant $U$ (c), as a function of the noise parameter $q$ for several values of the system size $N$. The critical point $q_{c}=0.034(8)$ is estimated as the point at which the different curves for different $N$ intercept each other.
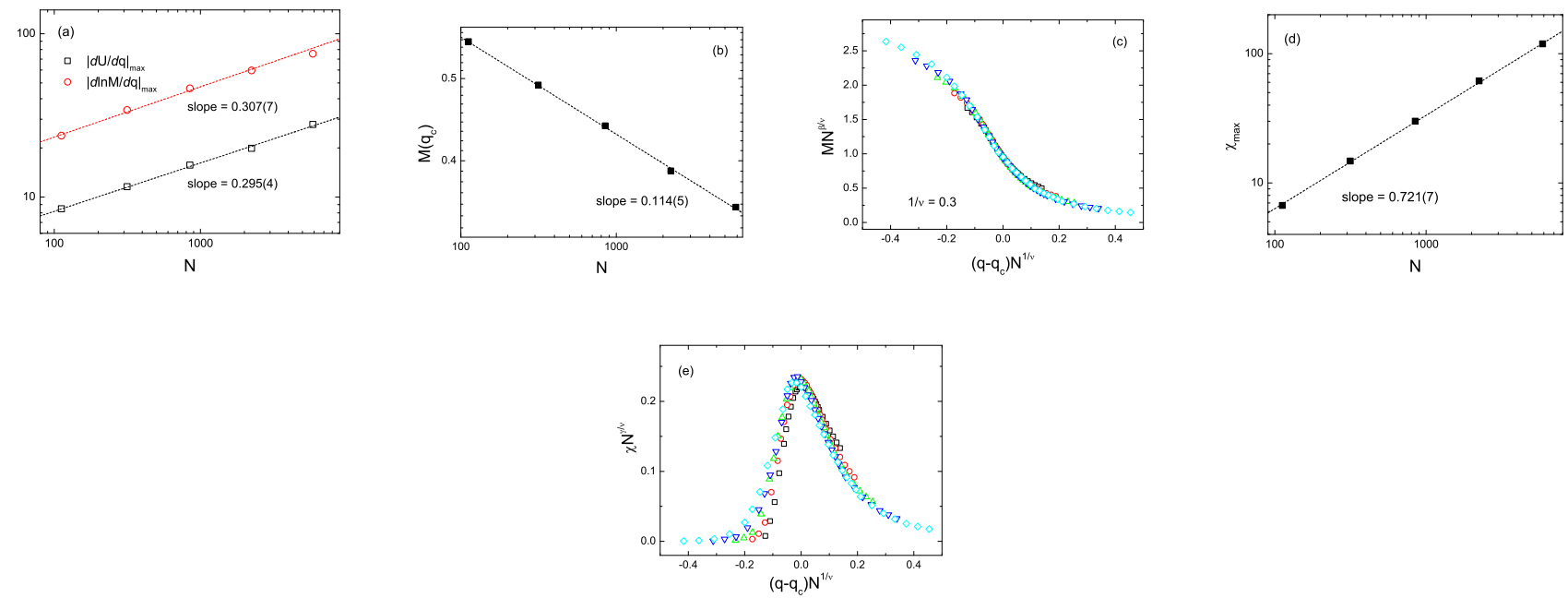

FIG. 5: (Color online) (a) Log-log plot of the size dependence of the maximum values of derivatives of various thermodynamic quantities used to determine $1 / \nu$. (b) $\log -\log$ plot of the magnetization at $q=q_{c}$ as a function of $N$. The slope of the best fit gives $\beta / \nu=0.114(5)$. (c) Data collapse of the magnetization $M$ shown in Fig. 4(a). The exponents used for the data collapse $1 / \nu=0.3, \beta / \nu=0.115$. (d) Log-log plot of the maximum of the susceptibility as a function of $N$. From this we estimate the critical exponent $\gamma / \nu=0.721(7)$ as the best fit of data points. (e) Data collapse of the susceptibility shown in Fig. 4(c). The fitting exponents $\beta / \nu=0.722,1 / \nu=0.3$.

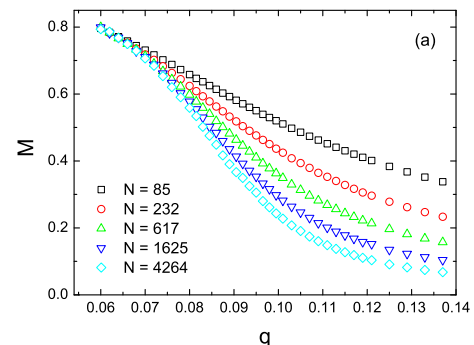

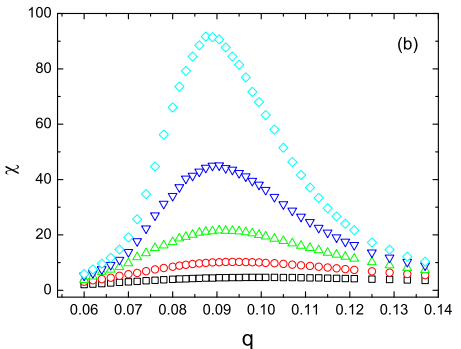

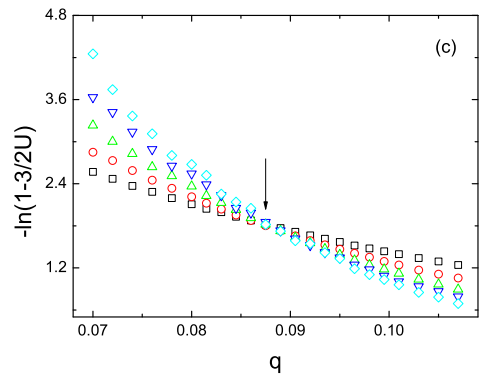

FIG. 6: (Color online) Majority-vote model on the dual heptagonal lattices. Magnetization $M$ (a), susceptibility $\chi$ (b), and reduced fourth-order cumulant $U$ (c), as a function of the noise parameter $q$ for several values of the system size $N$. The critical point $q_{c}=0.087(5)$ is estimated as the point where the curves for different $N$ intercept. 

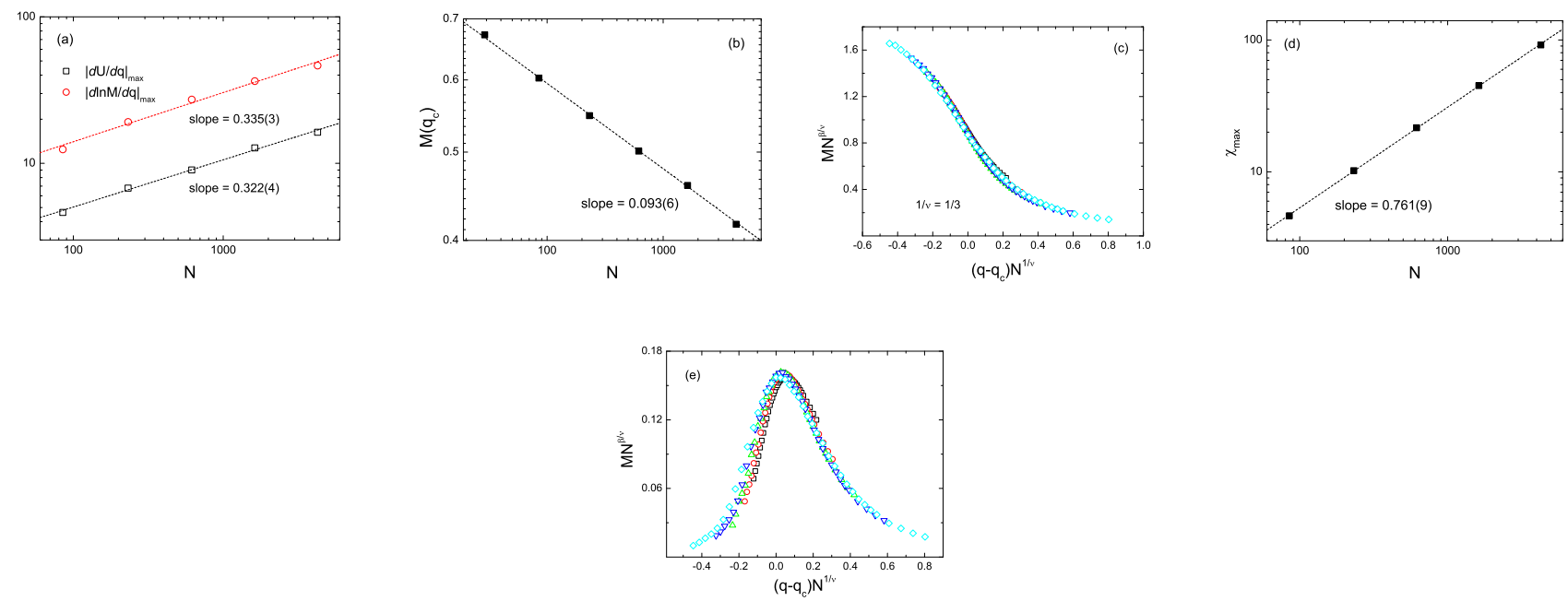

FIG. 7: (Color online) (a) Log-log plot of the size dependence of the maximum values of derivatives of various thermodynamic quantities used to determine $1 / \nu$. (b) $\log -\log$ plot of the magnetization at $q=q_{c}$ as a function of $N$. The slope of the best fit of the points gives $\beta / \nu=0.093(6)$. (c) Data collapse of the magnetization $M$ shown in Fig. 6 (a). The exponents used for the data collapse $1 / \nu=1 / 3, \beta / \nu=0.094$. (d) $\log -\log$ plot of the maximum of the susceptibility as a function of $N$. From this plot we estimate the critical exponent $\gamma / \nu=0.761(9)$. (e) Data collapse of the susceptibility shown in Fig. 6(c). The fitting exponents $1 / \nu=1 / 3, \beta / \nu=0.762$.

with a large size, and investigate the magnetization in the center and compare that with a heptagonal lattice of a smaller size. In particular, we perform simulations on the heptagonal lattice at level $7(N=5887)$, and track the magnetization in the inner part within level $3,4,5$, and 6 separately. Then we compare the results with the magnetization of Fig. 4(a). The same procedure is also done for dual heptagonal lattice at level $8(N=$ 4264), but within level 4, 5, 6, and 7 respectively. The numerical results are summarized in Figs. 8 and 9, We note that the nonvanishing boundary nodes play different roles in the ordering processes. As can be observed in Fig. 8, the magnetization in all the inner parts of a big heptagonal lattice are greater than that on a heptagonal lattice with same size, which indicates that the existence of a higher level of boundary can facilitate the ordering of the inner spins. This picture is changed, however, on the dual heptagonal lattice. In the subcritical region $q<q_{c}$, the boundary nodes promote the ordering process as before. In the supercritical region $q>q_{c}$, on the contrary, they impose opposite influence on the ordering process driving the system towards more disorder [Fig. 9]. A structural cause for this phenomenon is, we believe, the different local topologies of the two lattices. Looking at Fig. [1 the nodes one step from the boundary have fewer connections to the boundary in the heptagonal lattice than its dual (the path-length going from the periphery to the center, for systems of the same size, are longer). The boundary is also more indirectly coupled to the interior in the heptagonal lattice. The boundary nodes of the dual heptagonal lattice should thus have a stronger influence on the configuration of the interior (we hesitate to say "bulk properties" since the boundary is a finite fraction of the interior). We expect similar stronger boundary effects for dual heptagonal lattices also exists for other statistical spin models defined.

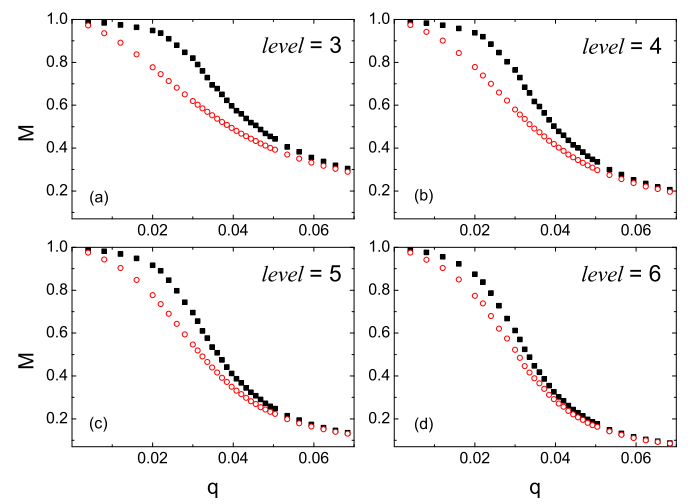

FIG. 8: (Color online) Magnetization $M$ as a function of the noise parameter $q$. (a) Filled squares represent the magnetization at the inner level 3 of the heptagonal lattice with 7 levels in total. Open circles represent the heptagonal lattice with 3 levels. (b), (c) and (d) show the same situation as (a) but for different levels. 

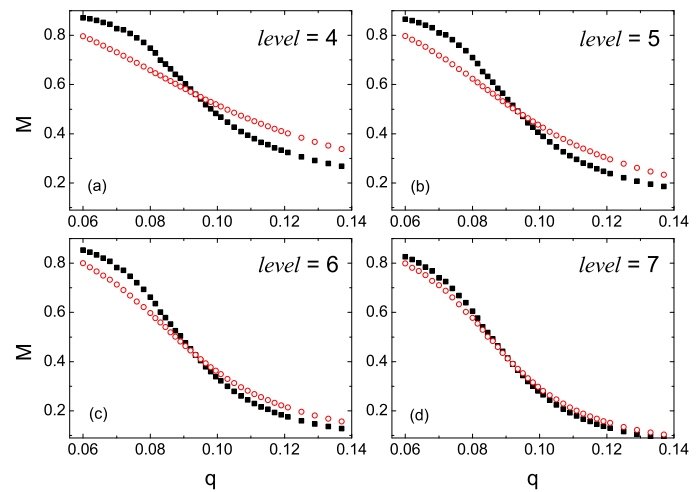

FIG. 9: (Color online) Magnetization $M$ as a function of the noise parameter $q$. (a) Filled squares symbolize the magnetization in the inner level 4 of the dual heptagonal lattice with 8 levels in total. Open circles represent the heptagonal lattice with 4 levels. (b), (c) and (d) show plots corresponding to (a) for other levels.

\section{E. Comparisons with the Ising model}

In Ref. 14], Oliveira found that the critical exponents for the majority-vote model on square lattice with periodic boundary condition are $\nu=0.99 \pm 0.05, \gamma / \nu=$ $1.73 \pm 0.05$, and $\beta / \nu=0.125 \pm 0.005$. These results demonstrate the majority-vote model on a torus has the same universal critical behavior as the equilibrium Ising model. Recently, Yang et al. investigated a slightly different majority-vote model on $d$-dimensional hypercubic lattices. Their model is identical to the original one by simple replacement of the temperature parameter to a noise parameter like in the present paper, $\tanh (1 / k T)=(1-2 q)$ [20]. Their simulation results suggest that the critical exponents for the majority-vote model in three dimensions are different from those of the Ising model, and that the results for four and five dimensions are far from the standard mean-field values. For their version of the majority-vote model in two dimensions, the global and local configuration energy differences between before and after spin-flipping are not identical 30], but the sign of both energies is the same, whereas for $d \geq 3$, the sign of the two types of energy differences is not always the same. For the Ising model, however, the differences of the global and local configuration energy are exactly identical, regardless of dimension. They conjectured that the discordance of the sign of the energy difference between the global and local energy is responsible for the different critical behaviors. Observe that our critical exponents $1 / \nu \approx 0.3$ and 0.33 in Fig. 5 (a) and Fig. 7(a), which are smaller than 0.5, so from the hyperscaling relation $\nu d=1$ ( $d$ is the dimension) we see that our hyperbolic lattices can be described as having an effective dimensionality greater than 2 , but smaller than 4 . In this sense, our present work provide comple- mentary support for the results obtained in Ref. [20]. In another recent work [1], Shima et al. studied the Ising model on heptagonal lattices, and found the critical exponent $\gamma / \nu=0.655$. Since our critical exponent $\gamma / \nu$, also considering the estimated error, is quite different from this value, we believe that the majority-vote model on the heptagonal lattice belongs to a different universality class as the Ising model on this topology. This result can be regarded as another evidence for the conjecture by Yang et al. 20] mentioned above.

\section{CONCLUSIONS}

To summarize, we have studied the critical behavior of the majority-vote model on the heptagonal and dual heptagonal lattices. These lattices possess a peculiar property: the ratio of the size of the boundary to the total size remains finite even in the thermodynamic limit. Finite-size scaling analysis reveals that the critical exponents for magnetization and susceptibility deviate from those of the majority-vote model on a torus (which belongs to the same universality class as the equilibrium Ising model) and are also different from those of the Ising model on heptagonal and dual heptagonal lattices. In particular, the best fit of these exponents provided $\beta / \nu=0.114(5), \gamma / \nu=0.721(7)$ for the heptagonal lattice, and $\beta / \nu=0.093(6), \gamma / \nu=0.761(9)$ for the dual heptagonal lattice. By comparing to the majority-vote model on square lattices with free boundary conditions, we found that the free boundaries result in strong finitesize scaling effect, which in turn leads to the measured effective dimensionality smaller than unity. Nonetheless, we believe that the critical exponents of the majorityvote model defined on negatively curved surface also satisfy the hyperscaling relation $2 \beta / \nu+\gamma / \nu=D_{\text {eff }}$.

Furthermore, for the hyperbolic lattices, we also investigated the effect of the boundary nodes on the ordering process. It was shown that the boundary nodes have different functions in our two lattices. For the heptagonal lattice, the boundary has a positive influence on ordering, whereas for the dual heptagonal lattice the boundary nodes can either facilitate or inhibit ordering depending on the magnitude of the noise. These findings are further evidence that the underlying geometric structure determine the critical properties of the majority-vote model. For the future, it will be interesting to explore the effect of boundary nodes on the ordering processes of other statistical spin models defined on hyperbolic lattices.

\section{Acknowledgments}

The authors wish to thank Dr. Seung Ki Baek for numerous valuable comments and suggestions. Z.X.W. acknowledges financial support from the Swedish Research Council. P.H. acknowledges support from the Swedish Foundation for Strategic Research, the Swedish Research 
Council and the WCU (World Class University) program through the Korea Science and Engineering Foundation funded by the Ministry of Education, Science and Technology (R31-2008-000-10029-0).
[1] H. Shima and Y. Sakaniwa, J. Phys. A 39, 4921 (2006).

[2] H. Shima and Y. Sakaniwa, J. Stat. Mech. (2006) P08017.

[3] R. Krcmar, T. Iharagi, A. Gendiar, and T. Nishino, Phys. Rev. E 78, 061119 (2008).

[4] Y. Sakaniwa and H. Shima, Phys. Rev. E 80, 021103 (2009).

[5] S. K. Baek, P. Minnhagen, and B. J. Kim, EPL 79, 26002 (2007).

[6] S. K. Baek, H. Shima, and B. J. Kim, Phys. Rev. E 79, 060106 (2009).

[7] W. A. Moura-Melo, A. R. Pereira, L. A. S. Mól, and A. S. T. Pires, Phys. Lett. A 360, 472 (2007).

[8] A. Gendiar, R. Krcmar, K. Ueda, and T. Nishino, Phys. Rev. E 77, 041123 (2008).

[9] S. K. Baek, P. Minnhagen, H. Shima, and B. J. Kim, Phys. Rev. E 80, 011133 (2009).

[10] S. K. Baek, P. Minnhagen, and B. J. Kim, Phys. Rev. E 79, 011124 (2009).

[11] S. K. Baek, S. D. Yi, and B. J. Kim, Phys. Rev. E 77, 022104 (2008).

[12] N. Park, M. Yoon, S. Berber, J. Ihm, E. Osawa, and D. Tománek, Phys. Rev. Lett. 91, 237204 (2003).

[13] H. S. M. Coxeter, Introduction to Geometry (Wiley, New York, 1969).

[14] M. J. de Oliveira, J. Stat. Phys. 66, 273 (1992).

[15] M. J. de Oliveira, J. F. F. Mendes, and M. A. Santos, J. Phys. A 26, 2317 (1993).

[16] M. C. Marques, J. Phys. A 26, 1559 (1993).

[17] M. A. Santos and S. Teixeira, J. Stat. Phys. 78, 963 (1995).

[18] G. Grinstein, C. Jayaprakash, and Y. He, Phys. Rev. Lett. 55, 2527 (1985).
[19] W. Kwak, J.-S. Yang, J.-I. Sohn, and I.-M. Kim, Phys. Rev. E 75, 061110 (2007).

[20] J.-S. Yang, I.-M. Kim, and W. Kwak, Phys. Rev. E 77, 051122 (2008).

[21] F. W. S. Lima, U. L. Fulco, and R. N. Costa Filho, Phys. Rev. E 71, 036105 (2005).

[22] L. F. C. Pereira and F. G. B. Moreira, Phys. Rev. E 71, 016123 (2005).

[23] F. W. S. Lima, A. O. Sousa, and M. A. Sumuor, Physica A 387, 3503 (2008).

[24] P. R. A. Campos, V. M. de Oliveira, and F. G. Brady Moreira, Phys. Rev. E 67, 026104 (2003).

[25] F. W. S. Lima, Int. J. Mod. Phys. C 17, 1257 (2006); F. W. S. Lima and K. Malarz, Int. J. Mod. Phys. C 17, 1273 (2006).

[26] D. Stauffer and K. Kuĺakowski, J. Stat. Mech. (2008) P04021.

[27] V. Privman, Finite-Size Scaling and Numerical Simulation of Statistical Systems (World Scientific, Singapore, 1990).

[28] P. Holme and M. E. J. Newman, Phys. Rev. E 74, 056108 (2006).

[29] A. M. Ferrenberg and D. P. Landau, Phys. Rev. B 44, 5081 (1991); W. Janke, M. Katoot, and R. Villanova, Phys. Rev. B 49, 9644 (1994); K. Binder and E. Luijten, Phys. Rep. 344, 179 (2001).

[30] The local configuration energy in Ref. [20] is defined as $E\left[\sigma_{i}\right]=-\sigma_{i} S\left(\sum_{j \in \Omega_{i}} \sigma_{j}\right)$, where $S(x)$ and $\Omega_{i}$ are the same as defined in Eq. (1). The global configuration energy is obtained from summing the local configuration energy over all the sites. 